\title{
Benchmark Analysis of the HTR-10 with the MAMMOTH Reactor Physics Application
}

Javier Ortensi, Sebastian Schunert, Yaqi Wang, Vincent Labouré, Frederick Gleicher, Richard C. Martineau

Nuclear Science and Technology Directorate

Idaho National Laboratory

P.O. Box 1625

Idaho Falls, ID 83415-3840

June 2018

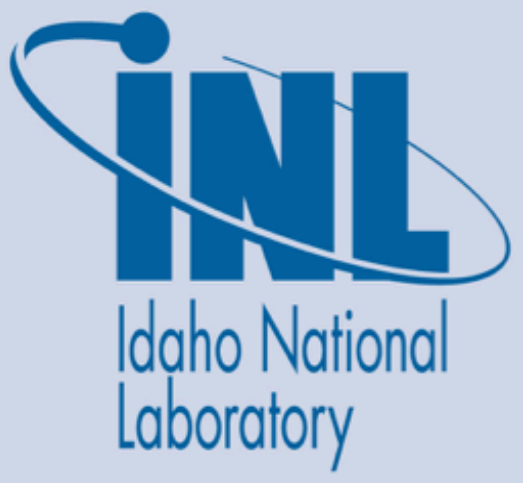

INL is a U.S. Department of Energy National Laboratory operated by Battelle Energy Alliance 


\section{NOTICE}

This information was prepared as an account of work sponsored by an agency of the U.S. Government. Neither the U.S. Government nor any agency thereof, nor any of their employees, makes any warranty, expressed or implied, or assumes any legal liability or responsibility for any third party's use, or the results of such use, of any information, apparatus, product, or process disclosed herein, or represents that its use by such a third party would not infringe on privately owned rights. The views expressed herein are not necessarily those of the U.S. Nuclear Regulatory Commission. 


\title{
Benchmark Analysis of the HTR-10 with the MAMMOTH Reactor Physics Application
}

\author{
Javier Ortensi, Sebastian Schunert, Yaqi Wang, Vincent Labouré, Frederick
} Gleicher, Richard C. Martineau

June 2018

Idaho National Laboratory

Nuclear Science and Technology Directorate

Idaho Falls, Idaho 83415

http://www.inl.gov

Prepared for the

Office of Nuclear Regulatory Research

U. S. Nuclear Regulatory Commission

Washington, D. C. 20555

Task Order No.: NRC-HQ-60-17-T-0011 


\section{Executive Summary}

This report documents a set of benchmarks used to validate the Serpent and MAMMOTH neutron transport models of the HTR-10 reactor developed at Idaho National Laboratory. The high-fidelity Serpent Monte Carlo models of the HTR-10 critical and full core configurations include both random, discrete distributions of TRISO particles in the pebbles and random, discrete pebble distributions in the pebble bed core. The Serpent results agree very well with the critical and control rod worth measurements provided in the International Reactor Physics Experiment Evaluation Project (IRPhEP) report if the ENDF/B-VII.r1 dataset is used. These Serpent models are subsequently used to prepare cross sections and flux tallies in a 10 coarse energy group structure for the MAMMOTH Reactor Physics MOOSEbased application. We find that MAMMOTH can reproduce the Monte Carlo solution for the HTR-10 reactor by using the PJFNK-SPH equivalence method. In all cases studied, the MAMMOTH results are within $120 \mathrm{pcm}$ of the Monte Carlo reference calculation and the maximum errors in neutron absorption and generation rates are within $0.536 \%$ and $0.215 \%$ from the reference calculation, respectively. A set of benchmark exercises from the International Atomic Energy Agency (IAEA) HTR-10 benchmark in IAEA-TECDOC-1382 were conducted including parts B1 (critical), B2 (temperature coefficient) and B3 (control rod worth). The results we obtain for the critical core are in excellent agreement with the expected value and we confirm that with ENDF/B-VII.r1 data we match the critical eigenvalue $\mathrm{keff}=1$ to within $100 \mathrm{pcm}$. Our temperature coefficient calculation is consistent with the VSOP results generated by the German participants. The Chinese and South African VSOP models produce lower temperature coefficients, a fact that was noted in the original IAEA benchmark, but for which no explanation was provided. Both a full and single control rod worth calculations with Serpent and MAMMOTH agree well with the results from the IAEA benchmark. Finally, and most importantly, this work demonstrates that MAMMOTH can recover the Monte Carlo high-fidelity solutions to an excellent accuracy using the PJFNK-SPH method. This sets the stage for the ultimate goal of using MAMMOTH in transient, multiphysics scenarios where correction of the cross sections is obtained for steady-state conditions and then used in the transient scenario for which no Monte-Carlo solution is available. 


\section{Contents}

$\begin{array}{lll}1 & \text { Introduction } & 1\end{array}$

2 Computer Codes and Methods 5

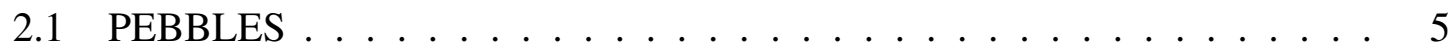

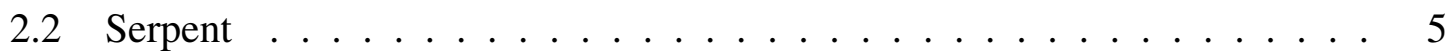

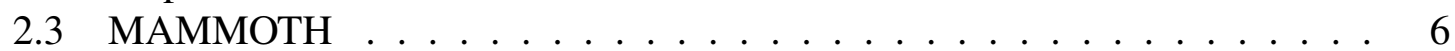

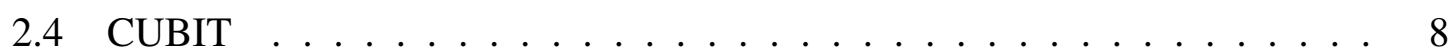

3 Models 9

$3.1 \quad$ Pebble Distributions. . . . . . . . . . . . . . . . . . . . . . . . . . . . . 9

3.2 Cross Section Preparation $\ldots \ldots \ldots \ldots$

3.3 Mesh Generation . . . . . . . . . . . . . . . . . . . . . . 16

3.4 Deterministic Neutronics Solution $\ldots \ldots \ldots \ldots \ldots$

4 Results 21

4.1 Benchmark Problem B1: Initial Criticality . . . . . . . . . . . . . 21

4.2 Benchmark Problem B2: Temperature Coefficient of Reactivity. . . . . . . 23

4.3 Benchmark Problem B3: Control Rod Worth $\ldots \ldots \ldots$. . . . . . 27

$\begin{array}{lll}5 \text { Conclusion } & 31\end{array}$

6 Future Work 33 


\section{List of Figures}

$1 \quad$ HTR-10 geometry with vessel. . . . . . . . . . . . . . . . 3

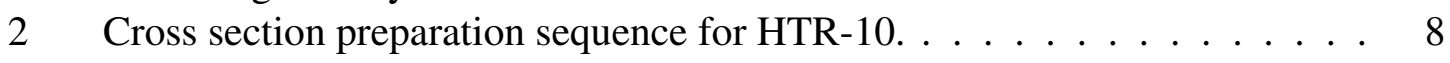

$3 \quad$ Pebble distribution for the full core configuration. . . . . . . . . . . . 10

$4 \quad$ TRISO distribution in a pebble distribution $\ldots \ldots \ldots \ldots . \ldots 11$

$5 \quad$ XY view of the HTR-10 full core Serpent model. . . . . . . . . . . . . 12

$6 \quad$ YZ view of the HTR-10 critical and full core Serpent models . . . . . . . . 13

7 Important cross sections for HTR-10 calculations. . . . . . . . . . . . . . 14

$8 \quad$ Macroscopic cross sections as a function of radial position. . . . . . . . . 15

$9 \quad$ Homogenization region assignment for HTR-10. $\ldots \ldots \ldots \ldots$

$10 \quad$ Flux distribution in the critical core (axial centerline). . . . . . . . . . . . . 24

11 Diametric flux distribution for the one-rod-in configuration. . . . . . . . . 28

12 Scalar flux in group 10 for one-rod in configuration. . . . . . . . . . . . . 29

13 Comparison for scalar flux in group 10 for all three rod configurations. . . . 30 


\section{List of Tables}

$1 \quad$ Upper energy group boundaries $(\mathrm{eV}) . \ldots \ldots$. . . . . . . . . . . . . 14

2 Eigenvalues computed with different codes for the initial critical configu-

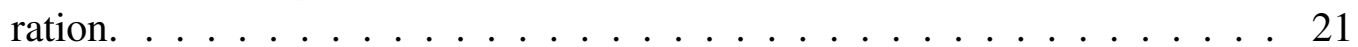

$3 \quad$ Single Control Rod Worth for the $17 \mathrm{k}$ initial critical core configuration. . . 22

$4 \quad$ Relative differences of eigenvalues (pcm) and reaction rates (\%) between MAMMOTH and the Serpent model for the initial critical core. . . . . . . 23

5 Eigenvalue computed with different codes at three temperatures for the full

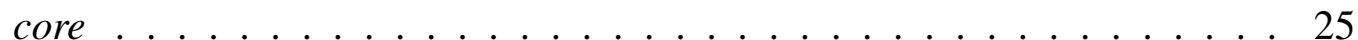

$6 \quad$ Isothermal Temperature Coefficient of Reactivity for the full core. . . . . . 26

$7 \quad$ Relative differences of eigenvalues (pcm) and reaction rates (\%) between MAMMOTH and Serpent for the full core under isothermal conditions. . . 26

$8 \quad$ Control Rod Worth (\%) for the full core configuration. . . . . . . . . . . . 27

9 Relative difference of control rod worth (\%) comparing MAMMOTH and

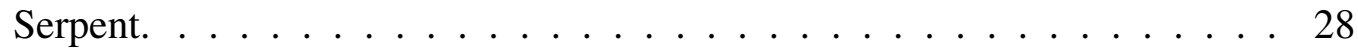




\section{Introduction}

This report records a feasibility study undertaken at Idaho National Laboratory (INL) for using the reactor multiphysics application MAMMOTH [1] in pebble-bed reactor (PBR) analysis based on two HTR-10 benchmarks [2, 3]. MAMMOTH is a unique reactor physics code that is built on the MOOSE Finite Element Method (FEM) framework and utilizes the Rattlesnake [4] radiation transport code. Its design allows seamless coupling with MOOSE modules containing heat conduction and solid mechanics functionality and with the thermal fluids code Pronghorn [5, 6]. This work is the first step in establishing the coupled capabilities of MAMMOTH, Pronghorn and other applications within the MOOSE-based applications as high fidelity tools for transient, multiphysics PBR analysis.

The HTR-10 is a small pebble-bed test reactor rated at a thermal power of $10 \mathrm{MWt}$ intended as a stepping stone for the development of PBR technology in China. HTR-10 is located at the Institute of Nuclear and New Energy Technology (INET) and has achieved initial criticality on 1 December 2000. The design of the HTR-10 reactor represents the design features of the modular High-Temperature Gas-cooled Reactor (HTGR) which is primarily characterized by inherent safety features. The reactor geometry is depicted in Fig. 1. The following discussion of the reactor geometry and the benchmark based thereon are taken from [2]. This reference discusses the initial critical and a 17,000 pebble core configuration of HTR-10, herein referred to as the $17 k$ core. The initial criticality was achieved with the initial critical configuration while control rod worth measurements were performed with the $17 k$ configuration that has 110 pebbles more in the core and hence features a small difference in excess reactivity.

Overall, the neutronically relevant core, reflector and shielding regions (everything inside of the boronated carbon bricks and carbon bricks in Fig. 1) are 6.1 meters tall and have a radius of 1.9 meters; the core containing the pebble bed is split into the upper part containing the cylindrical core and the upper cone; and a lower part containing the lower cone and the discharge tube. The upper part of the core during the initial critical contains a mix of 16,890 fuel and "dummy" graphite pebbles with a ratio of $57: 43$, while the lower part of the core only contains dummy pebbles. The benchmark report assumes a uniform packing fraction of 0.61 throughout the whole core. The control rod borings, as well as the cold coolant flow channels are depicted in Fig. 1 .

In addition to the initial critical configurations, Ref. [3] describes the full core configuration that features a significantly larger number of pebbles in the upper core region. This full core configuration is attained by loading more pebbles starting from the initial critical 
configuration until the core is capable of being operated at full power. The volume of the fuel pebbles in the upper core region is estimated to be $5 \mathrm{~m}^{3}$; at a packing fraction of 0.61 and a pebble radius of $3 \mathrm{~cm}$, this corresponds to 26,992 pebbles in the upper core region.

Within this work, we focus on a subset of benchmark exercises described in Ref. [3]. First, a modified version of the benchmark exercise B1 of the initial critical core is performed: instead of finding the loading that leads to a critical core, we use the description in Ref. [2] specifying the number of loaded pebbles $(16,890)$. Further, benchmark exercises B2 and $\mathrm{B} 3$ are performed and we report effective multiplication factors at $20^{\circ} \mathrm{C}, 120^{\circ} \mathrm{C}$ and $250^{\circ} \mathrm{C}$, the isothermal temperature coefficients of reactivity in those ranges and the control rod worth of all rods at $20^{\circ} \mathrm{C}$. Finally, for benchmark exercise B32, we report control rod worth at $20^{\circ} \mathrm{C}$ for a single inserted control rod with all other control rods being in the full out position. Reference [3] does not clearly define the control rod worth, but there are several indications in the sections from the Russian and French participants that the equation for the calculation of the reactivity worth was

$$
\rho=\frac{\left|k_{\text {in }}-k_{\text {out }}\right|}{k_{\text {out }}} \times 100 \%
$$

where $\mathrm{k}_{\text {in }}$ and $\mathrm{k}_{\text {out }}$ are the multiplication factors obtained for the rod in and rod configurations, respectively. In this work, we will report both this reactivity and the traditional definition of rod worth $(\Delta \rho)$ with

$$
\Delta \rho=\frac{\left|k_{\text {in }}-k_{\text {out }}\right|}{k_{\text {in }} k_{\text {out }}} \times 100 \%,
$$

since for numerical results above the critical state (1.0000) Equation 1 can give differences of several tenths of percent in rod worth.

This report is organized as follows: section 2 introduces the computational methods and tools used for performing this work, section 3 discusses the deployed models and relevant simplifying assumptions, section 4 presents the results obtained for this work, sections 5 and 6 draw conclusions and state future directions of work to establish MAMMOTH as a high-fidelity tool for transient, multiphysics PBR analysis. 


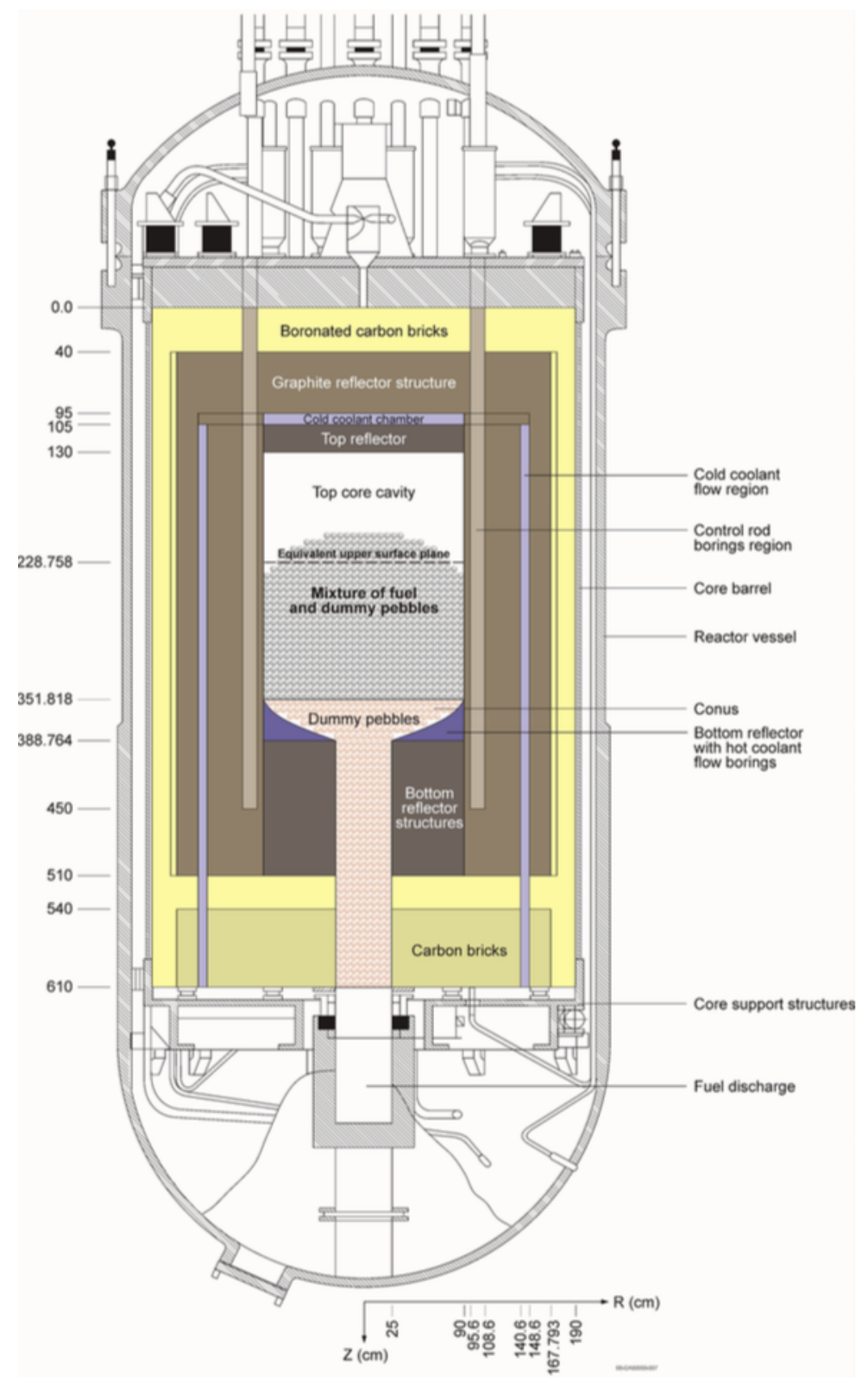

Figure 1: HTR-10 geometry with vessel (picture courtesy [2]). 


\section{Computer Codes and Methods}

The various computer codes and methods used in the calculations provided in this report are included in this section. The discussion will center around the PEBBLES code [7], used in the calculation of the pebble packing fractions, the Serpent code [8] for cross section generation and reference solutions, the MAMMOTH code [1] for reactor physics and the CUBIT code [9] for mesh generation.

\subsection{PEBBLES}

PEBBLES [7] is a discrete element method (DEM) code developed at INL to solve the equations of motion for a collection of pebbles in mechanical contact with each other and with external walls. The code simulates gravitational force and contact forces including kinetic and true static friction. For each pebble, the force and torque balances are used to compute the linear and angular acceleration that are integrated from suitable initial conditions to yield the trajectory of each pebble. The interaction of pebbles with each other is modeled using Hooke's law for normal forces and true static friction allowing non-zero angles of repose for tangential forces.

PEBBLES has been used for a variety of tasks including simulation of the effect of earthquakes on a PBR [10], calculation of packing fractions, Dancoff factors, pebble wear [11] and the pebble force on the walls. PEBBLES was originally used in the IRPhEP benchmark [2] to compute the pebble distribution in the HTR-10 critical core region above the conus, i.e. the cylindrical core region and the upper cone region. In this work, PEBBLES is employed in the computation of the pebble distribution for the full core configuration and includes all regions (conus and discharge chute).

The output of PEBBLES is a list of $N$ pebble center positions that are used by the downstream neutronics analysis tools. Post-processing Python scripts designate the fueled and non-fueled pebbles using a randomization algorithm to achieve the correct ratio of $57: 43$ for both the critical and the full core configurations.

\subsection{Serpent}

Serpent [8] is a three-dimensional continuous-energy Monte Carlo reactor physics code developed at VTT Technical Research Centre of Finland. It was selected as the main cross 
section preparation tool for this project because it offers 3-D spatial homogenization and group constant generation for deterministic reactor simulator calculations. At the same time, Serpent 2 provides a detailed reference calculation without energy, angular, or spatial discretization error. The version used in this work is 2.1.28.

Serpent has unique capabilities with regard to the modeling of PBRs. Serpent can not only model the random pebble distribution, but, in addition, it can model the random TRIstructural-ISOtropic (TRISO) distribution inside the pebbles. Serpent only requires a file with the position of each pebble's center point, much like the output generated by PEBBLES. Serpent uses this information to explicitly represent the location of pebbles in the core without applying any homogenization.

Serpent has been used at the INL to prepare neutron cross sections since 2010 [12]. Recent work with modeling and simulation of the TREAT reactor [13, 14] has improved the process for the preparation and tabulation of multi-group cross sections from Serpent to the MAMMOTH reactor physics application, thus making it a compelling candidate for this task.

\section{3 МАMМOTH}

The HTR-10 benchmark exercise requires the computation of core eigenvalues in the absence of multiphysics feedback, i.e. at uniform, constant steady-state conditions. The relevant physics describing the system under these conditions is the neutron transport equation in its steady state eigenvalue form [4]. In this report we omit stating the transport equation and refer the reader to Ref. [4] for a thorough discussion; it it sufficient to say that the neutron transport equation requires cross sections describing the interaction of neutrons with the reactor material.

The MAMMOTH [1] reactor multiphysics analysis application developed at INL is used for computing the core multiplication factor and power distribution. MAMMOTH is based on MOOSE [15], a finite element method framework that focuses on multiphysics simulations with strongly or tightly coupled physics applications. In addition to implementing reactor physics specific capabilities such as depletion and equivalence methods, MAMMOTH seamlessly interfaces with several other MOOSE applications including Rattlesnake [16] for radiation transport algorithms, MOOSE modules for heat transfer and solid mechanics [15], BISON [17] for fuel performance modeling and Pronghorn [5, 6] for thermal fluids calculations. The Rattlesnake neutron transport solver incorporates a variety of spa- 
tial and angular discretization methods including diffusion, $P_{N}$ and $S_{N}$ (both $1^{\text {st }}$ and $2^{\text {nd }}$ order formulations). In this work, we exclusively use the second order $P_{N}$ discretized with continuous FEM and the continuous FEM diffusion solver. In addition, the computation of directional diffusion coefficients requires the solution of a neutron-transport like equation that is facilitated with the first order $S_{N}$ solver. A detailed discussion of the available transport discretization options in Rattlesnake can be found in Ref. [4].

Between the top of the pebble bed core and the top reflector, HTR-10 contains a cavity region that is filled with ambient, moist air. Neutronically, this region is optically transparent supporting significant streaming and a very small probability of collisions for neutrons traversing it. This traditionally poses problems for obtaining diffusion coefficients because the validity of the classical diffusion approximation for neutron transport applications ultimately requires short mean free paths and many scattering collisions before absorption; this is evident in the standard definition of the diffusion coefficient as the inverse of three times the transport cross section [18]. For optically thin regions, the diffusion coefficient tends to infinity. A variety of methods exists to meaningfully define diffusion coefficients in nearvoid regions, e.g. Morel's non-local diffusion coefficients [19] that are successfully used by Trahan [20] for analysis of reactors with optically thin channels, Monte-Carlo methods have been equipped with tallies to compute diagonal tensor diffusion coefficients based on the cumulative migration method [21]. Serpent currently does not include the capability to generate anisotropic diffusion coefficients in extended geometries to allow better modeling of neutron streaming effects within the diffusion approximation and hence we resort to using Morel's non-local diffusion coefficient in the top core cavity.

The computation of non-local diffusion tensors was implemented in the Rattlesnake transport solver [20, 14] to address the streaming effects through the region above the pebble bed. In addition to computing tensor diffusion coefficients, an advanced implementation of the traditional equivalence procedure [22] developed at INL, the PJFNK-SPH [23], is employed to ensure preservation of the reaction rates between the reference Monte Carlo model and the cross section set used in the MAMMOTH model. The final data preparation sequence presented in Figure 2 entails the generation of various cross section tabulations with Serpent, followed by the calculation of anisotropic diffusion coefficients and, finally, the equivalence correction of the cross sections with SPH. Rattlesnake automatically generates a new tabulation after each step without the need to use multiple datasets or other cumbersome data processing.

For the deterministic transport model, we do not explicitly model the location of all pebbles separately even though this capability is available in MAMMOTH through the pebble tracking transport algorithm implemented in Rattlesnake [24]. Instead, we infer the outline 


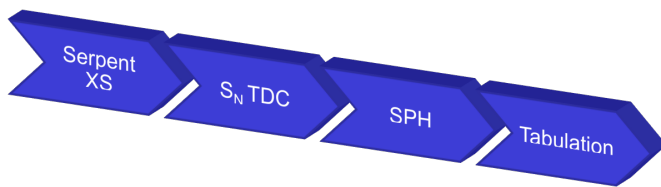

Figure 2: Cross section preparation sequence for HTR-10.

of the cylindrical core region and the top cone from the point cloud provided by PEBBLES and homogenize the top core cavity and the upper cone between $z_{c, 1}$ and $z_{c, 2}$ where $z_{c, 1}$ and $z_{c, 2}$ are the $\mathrm{z}$-coordinates of the lower onset of the top cone and the cone's tip.

\subsection{CUBIT}

Geometric models and their corresponding meshes were created with CUBIT, a full featured mesh generation toolkit designed by Sandia National Laboratory [9]. CUBIT was designed to create either structured or unstructured mesh elements in two- and threedimensions. CUBIT was initially built with the focus of utilizing quadrilateral and hexahedral meshes but has been expanded upon to include triangular and tetrahedral mesh elements. Algorithms within CUBIT have also been developed for generating continuous mixed element type meshes.

Finite element models for the purpose of this work were created in the EXODUS-II format. This format is used for efficient data storage and allows for problem definition, visualization and data extraction, and code to code data transfer within a singular mesh file [25]. Because of this capability, models for reactor analysis in MAMMOTH simulations are able to be performed on a single mesh file. 


\section{Models}

The geometry of the HTR-10 benchmark is specified in the benchmark report [3]. In the initial critical and full core configurations, fuel pebbles are only present in the cylindrical active core region, while the lower conus and discharge tube are filled with dummy pebbles. In the active core region, above the lower conus, a mix of fuel and dummy pebbles $(16,890$ at a ratio of $57: 43$ fuel to dummy pebbles) is loaded.

The initial critical core pebble distribution is adapted from the HTR-10 MCNP model used in the International Reactor Physics Experiment Evaluation Project [2]. We had no data for the full core configuration beyond the standard approximation for the pebbled-bed packing fraction of 0.61. We, therefore, develop a more realistic distribution with the PEBBLES code [7] for the full core HTR-10 active core, lower conus and discharge chute regions.

This section includes a description of the various models used in the calculations included in this report. The model used for the pebble distributions in the full core configuration is described in 3.1. A description of the Serpent Monte Carlo model and approximations, as well as the cross section preparation, is included in Section 3.2. The mesh generation is presented in section 3.3 .

\subsection{Pebble Distributions}

The geometric model in PEBBLES spans to the lower cone and chute regions. It does not, however, include the side dimples in the cylindrical vat. The results for the pebble distribution in the full core configuration are determined by two sequential PEBBLES calculations and are shown in Figure 3. In the first calculation, the re-circulation of a number of pebbles in the core is allowed for 20 seconds. The second calculation allows settling of the pebbles for an additional 20 seconds without re-circulation.

We use the stated core volume of $5.0 \mathrm{~m}^{3}$ and the packing fraction to compute the total number of pebbles in the active core region, i.e. above the lower conus:

$$
N_{a}=\frac{V_{a} \kappa}{\frac{4}{3} \pi r^{3}},
$$

where $N_{a}$ is the number of pebbles in the active core region, $V_{a}$ is the active core region, $\kappa$ is the packing fraction, and $r=3 \mathrm{~cm}$ is the pebble radius. We obtain $N_{a}=26,992$. It 
is noted that the total number of pebbles in the core, i.e. including lower conus and discharge tube is 31,275 because 2,126 and 2,157 pebbles are located in the bottom cone and discharge chute, respectively. The calculated value for $N_{a}$ is used for the PEBBLES runs. The PEBBLES calculated packing fraction is 0.6014 and hence slightly smaller than the benchmark value of 0.61 which follows observed average packing fractions of equilibrium cores. This discrepancy is expected because recirculation increases the packing fraction and hence we consider the slightly lower value obtained by PEBBLES to be more realistic. With a packing fraction of 0.6014 , the calculated core volume is roughly $V_{a}=5.07 \mathrm{~m}^{3}$.

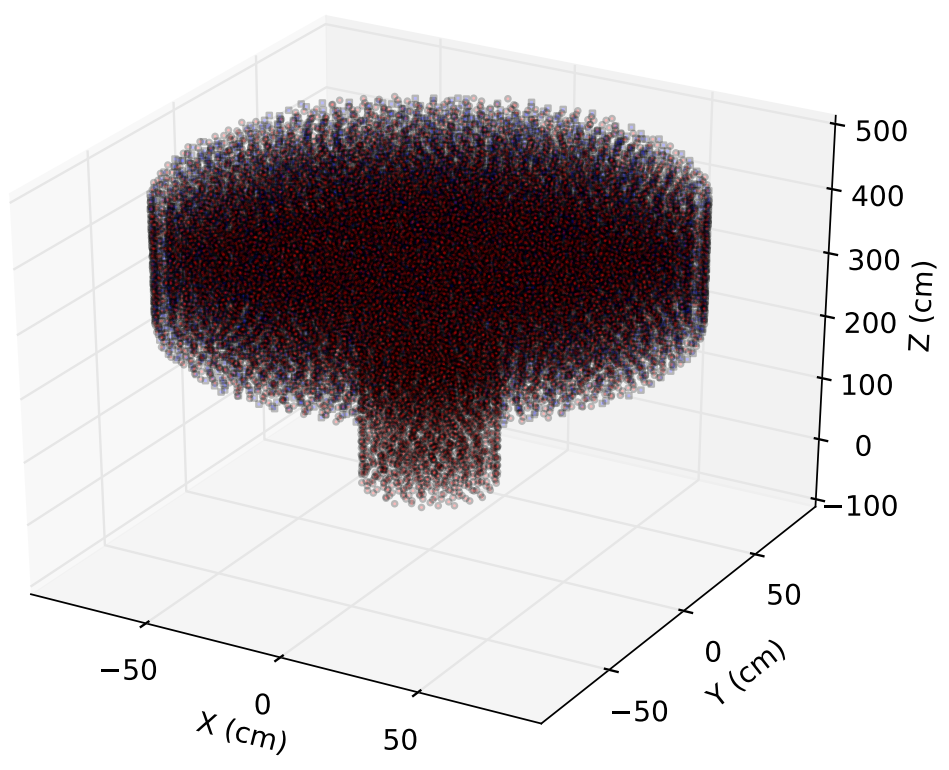

Figure 3: Pebble distribution for the full core configuration.

\subsection{Cross Section Preparation}

Geometry and material compositions of HTR-10 are based on the high-fidelity benchmark description included in [3] and [2]. The Serpent model used to generate multi-group cross sections uses PEBBLES computed distributions of the pebbles; the type of pebble - dummy 
or fuel - is assigned randomly to each pebble with probabilities corresponding to their relative abundance. Each pebble's interior structure is modeled as a random distribution of TRISO particles within the graphite ball. An example is shown in Figure 4.

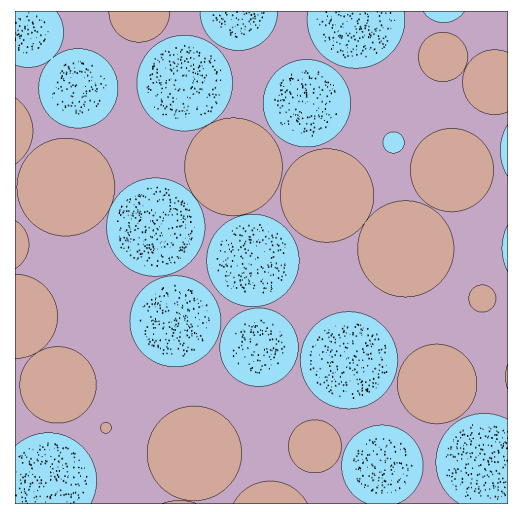

Figure 4: TRISO distribution in a pebble distribution. Fuel pebbles shown in blue and graphite "dummy" pebbles shown in brown.

The Serpent model for the critical core is very similar to the MCNP model developed in [2], with the following differences:

- the use of a random distribution of TRISO particles versus the original lattice constrained distribution in MCNP,

- the difference in the randomization of the fuel pebble assignment,

- approximating the KLAK (emergency reactivity control pebbles) channels as cylinders with an equivalent volume and

- the use of air instead of Helium for all calculations, adopted from Ref. [2].

The origin of the cross-section data for the majority of Serpent calculations in this report is the ENDF/B-VII.r1 evaluation. Some checks were also performed with the ENDF/B-VI data set to compare to the MCNP benchmark results.

The arrangement of dummy pebbles in the lower conus and discharge tube for the initial critical core is assumed to be Body-Centered Cubic (BCC). The BCC lattice parameter was adjusted to obtain a packing fraction of 0.61 , instead of the theoretical 0.68 . This is consistent with the initial MCNP model in Ref. [2]. 
The Serpent radial geometry is depicted in Fig. 5, its spatial extent is identical to the specified benchmark problems, i.e. it includes all regions depicted in Fig. 11 out to the boronated and carbon bricks (Note: the benchmark applies vacuum boundaries on the exterior of the boronated and carbon bricks, i.e. the colored volumes in Fig. 11. We observe a deterioration of the precision of tallies reporting thermal cross sections and fluxes for the boronated and carbon bricks at the bottom of the reactor. The deterioration of precision is caused by the low probability of neutrons streaming from the reactor core through the reflector and having an interaction of a specified type in the boronated or carbon brick region. Unless a significant number of Serpent histories are executed, Serpent judges the tallies as unreliable and reports zeroes for several thermal groups. The initial critical and fullcore configuration are further depicted in Fig. 6. The BCC arrangement in the lower conus is apparent in the initial critical configuration, while the full core configuration includes all of the pebbles.

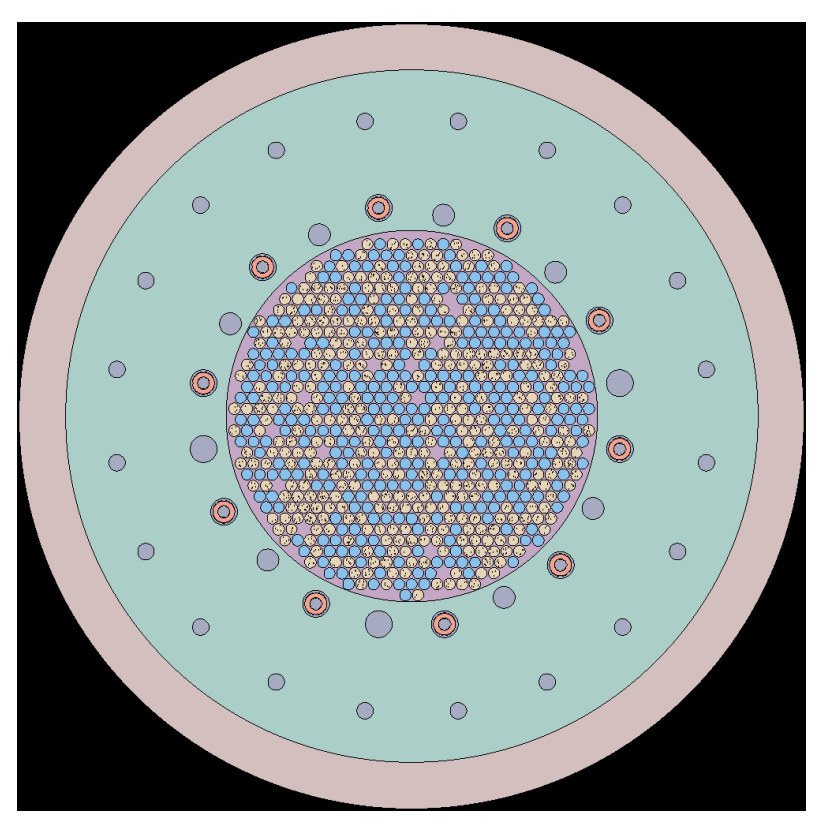

Figure 5: XY view of the HTR-10 full core Serpent model showing pebble-bed core in the center, control rod borings with control rods inserted, cylindrical KLAK channels (between control rods) and coolant channels. 


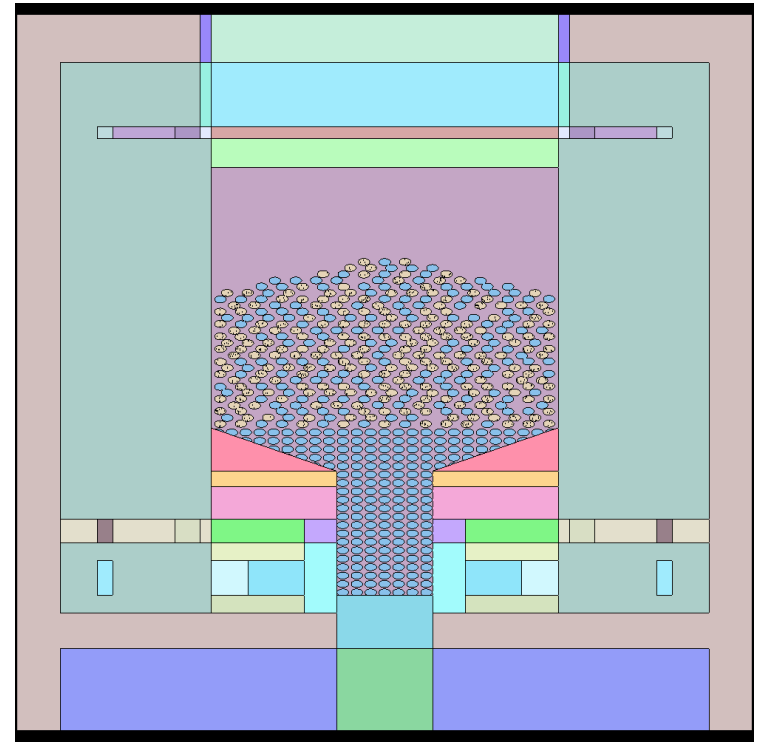

(a) initial critical

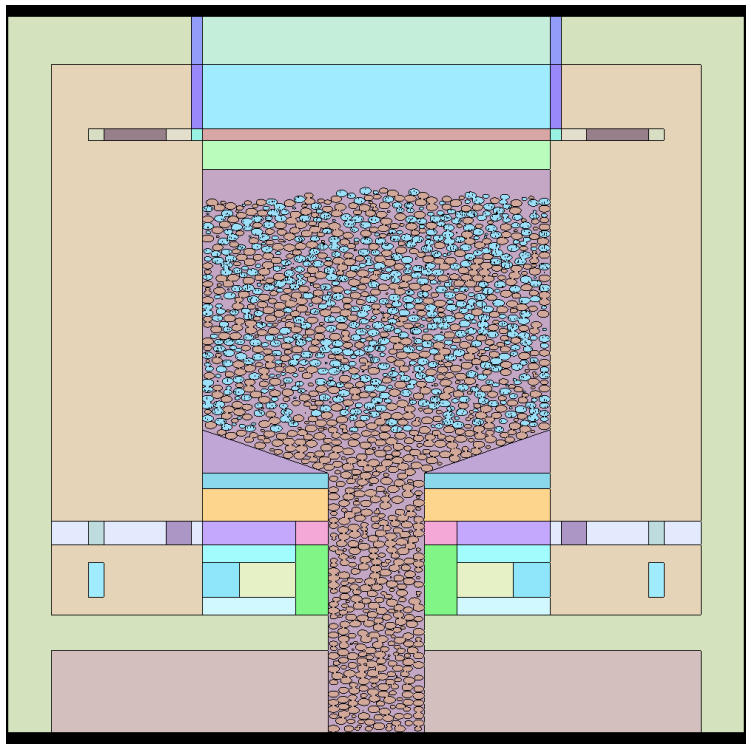

(b) full

Figure 6: YZ view of the HTR-10 critical and full core Serpent models. The BCC arrangement in the lower conus of the initial critical core is used to match the MCNP in Ref. [2]. The full core model includes all pebble positions from the PEBBLES run. Regions with different colors outside the active core indicate graphite with different impurities.

The Serpent cross sections are spatially homogenized over various material regions and energy-condensed to 10 energy groups; the energy group boundaries are listed in Table 1 . Spatial homogenization is facilitated in the FEM mesh generation process described in detail in section 3.3 by assigning both material and equivalence identifiers to intervals in the mesh generator's Constructive Solid Geometry (CSG) description. During the mesh generation process a Serpent formatted homogenization file is automatically created that can be directly included in the Serpent model.

The cross section generation for graphite moderated reactors with neutronically transparent coolants is substantially simpler than in other types of reactors. For the HTR-10 the active core region is the only region in the core with resonance absorbers like ${ }^{238} U$. In addition, the graphite cross section is quite straightforward since it transitions from a $1 / \mathrm{v}$ absorber to a constant for most of the energy range in this reactor. Figure 7 shows the various cross sections relevant to this reactor for fresh fuel. Both graphite and the ${ }^{10} B$ in the control rods are temperature independent and are not affected by spectral changes. Nevertheless, the changes in composition near homogenized control rod and coolant channels affect the 
Table 1: Upper energy group boundaries (eV).

\begin{tabular}{cc|cc}
\hline$g$ & $E_{g+1 / 2}$ & $g$ & $E_{g+1 / 2}$ \\
\hline 10 & 0.020 & 5 & 8.10 \\
9 & 0.047 & 4 & 132.7 \\
8 & 0.076 & 3 & 3481.1 \\
7 & 0.210 & 2 & 115620 \\
6 & 0.625 & 1 & $40 \mathrm{E} 6$ \\
\hline
\end{tabular}

number density of the materials, thus changing the macroscopic cross section. Only the active core cross sections near the reflector region will potentially experience spectral effects as the neutron energy spectrum can change in the presence of fuel.

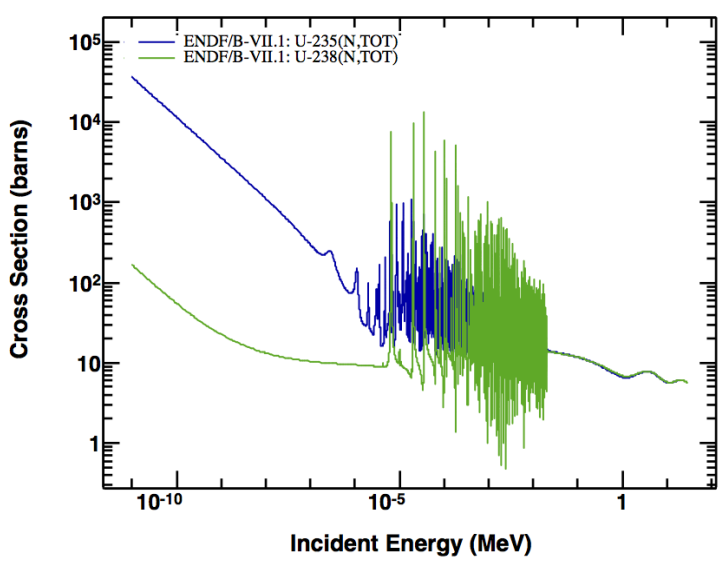

(a) resonance absorbers

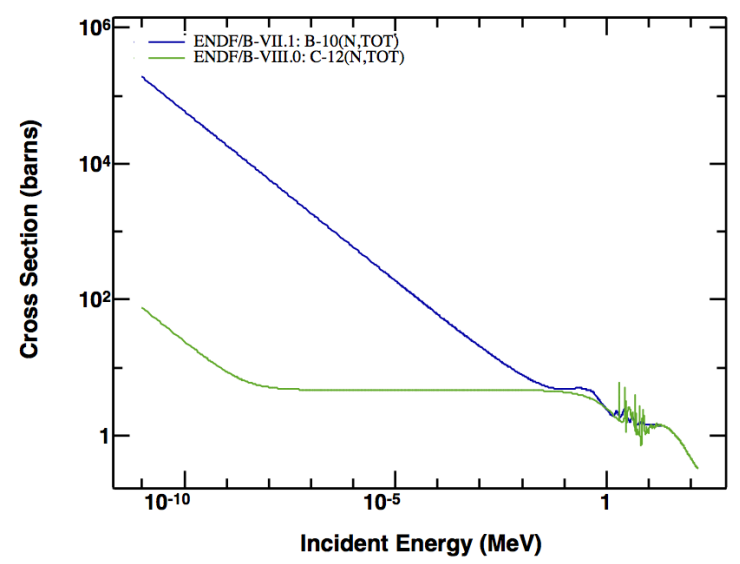

(b) $1 / \mathrm{v}$ absorbers

Figure 7: Important cross sections for HTR-10 calculations.

In order to illustrate some of the dependency of the cross sections, we used a 2-D core model, azimuthally homogenized, to evaluate the change in the cross sections as one moves radially away from the reactor centerline. Various cross sections as a function of radial position are plotted in Figure 8 . One can observe that the total cross section for this reactor core is dominated by the scattering cross section of graphite. A lower value of the scattering cross section is discernible near the active core edge at $85 \mathrm{~cm}$, where the number of pebbles decreases due to wall effects. Beyond the active core region, changes to the graphite cross section are due to changes in the material composition. Note that the boronated graphite 
bricks are not shown in these plots. Only the fastest energy group exhibits a significantly lower scattering and, consequently, total cross section, which is consistent with Fig. 7. The absorption cross section is nearly constant in the active core and reflector regions. Finally, the neutron production cross section experiences some wall effects and potential spectral effects at $85 \mathrm{~cm}$. In order to include this change in the cross sections, we divide cross sections zone in the core by including a $10 \mathrm{~cm}$ in the radial and axial directions in the vicinity of the reflector/wall regions.

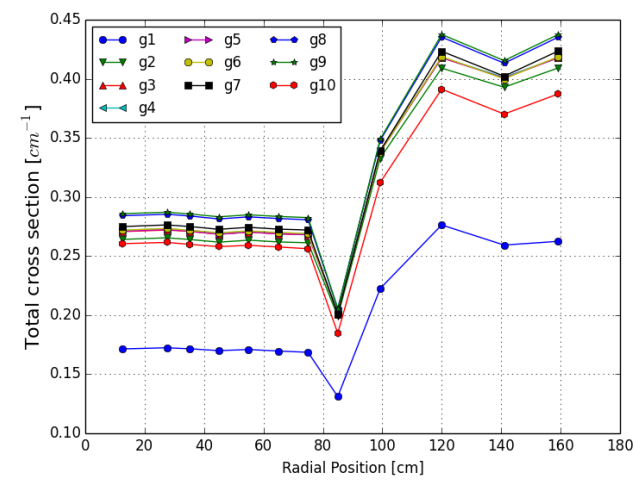

(a) Total

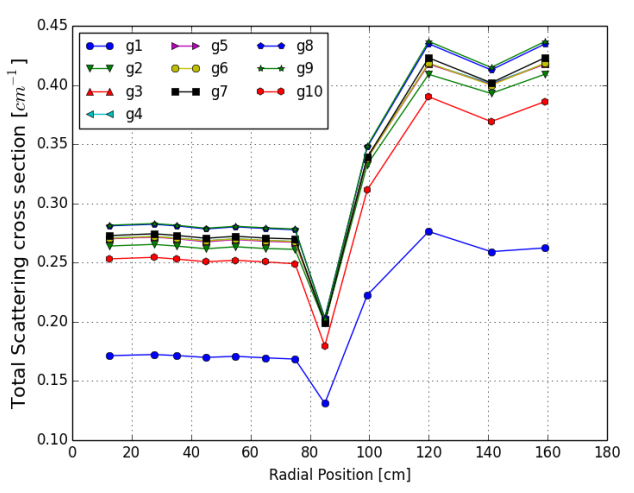

(c) Scattering

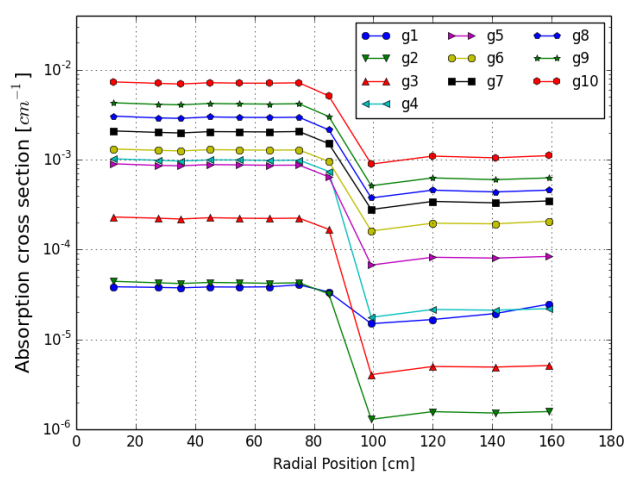

(b) Absorption

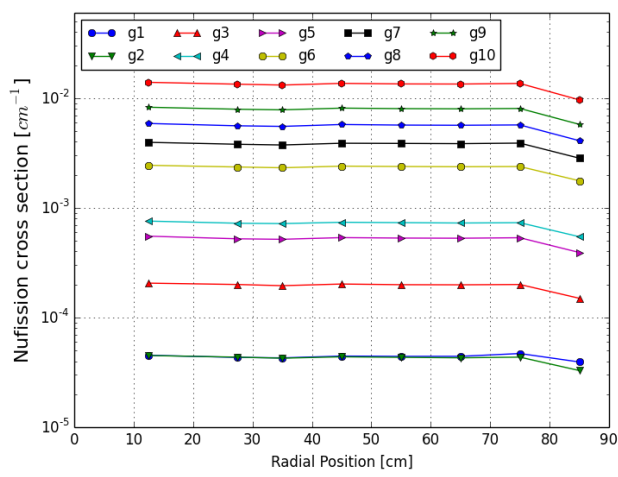

(d) Nufission

Figure 8: Macroscopic cross sections as a function of radial position. 


\subsection{Mesh Generation}

INL has developed a Python application that uses the CUBIT Python interface as a mechanism in which geometrical bodies and their associated meshes may be created and stored. It also provides an object-oriented structure that gives users the ability to easily manipulate and query bodies. The CSG input includes the general description of the PBR core as a set of concentric cylinders. It allows radial, axial and azimuthal refinement of the mesh as well as the assignment of material regions. The meshing scripts write both a material and equivalence (SPH) identification zone variables into the mesh in order to remove the cross section and equivalence zone assignment from the MAMMOTH input. The material IDs are specified by the user, but the equivalence zone assignment is automated and in a sequential order.

This Python application has been recently updated to generate an overlay mesh in cylindrical geometry that can be superimposed to any Serpent cylindrical model to match exactly the meshed geometry. The technology is easily extendable to Cartesian or hexagonal geometries. The advantage of this approach is to obtain homogenization instructions that exactly match the material regions in the MAMMOTH model hence reducing the need of human intervention. In contrast to creating homogenization instructions for Serpent the equivalence identifiers are used to create a set of flux tallies that are included in Serpent to generate region integrated reference fluxes throughout the geometry. We refer to the set of volumes with the same homogenization or equivalence identifiers as homogenization or equivalence regions, respectively. The sets of homogenization and equivalence regions can but do not need to be congruent. The homogenized cross section regions for HTR-10 are depicted in Fig.9.

The distinction between homogenization regions and equivalence regions was added to the MAMMOTH portfolio of capabilities. Usually tallying cross sections is more difficult than tallying fluxes so that small homogenization regions increase the computational burden on Serpent. In addition, certain material's nuclear cross sections such as Carbon are reasonably constant so that changes in the neutron spectrum do not lead to large changes in the obtained multi-group constants. Conversely, equivalence regions should be chosen as small as possible to ensure matching of reaction rates over more sub-regions of the geometry. In previous studies we have observed significant increase in accuracy with decreasing the size of the equivalence regions. Splitting homogenization and equivalence regions serves the primary purpose of increased accuracy while reducing the required amount of computational resources. 


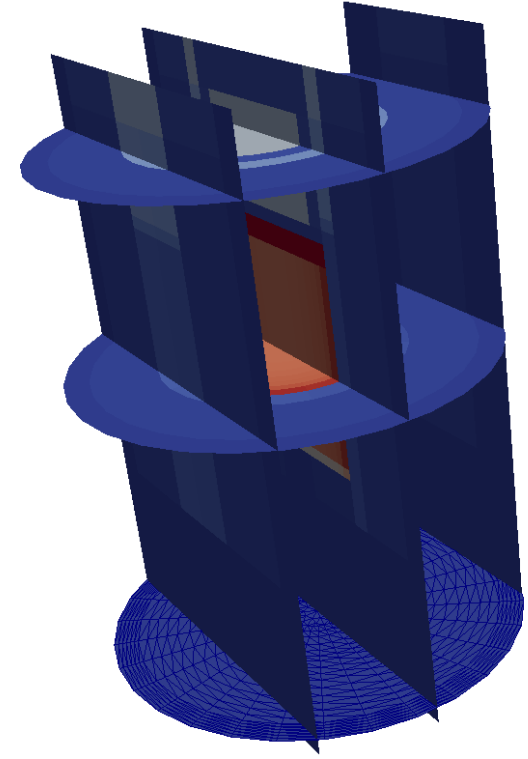

(a) Cut-away view of material identifier assignment for various layers normal to the $x$ and $z$ axes.

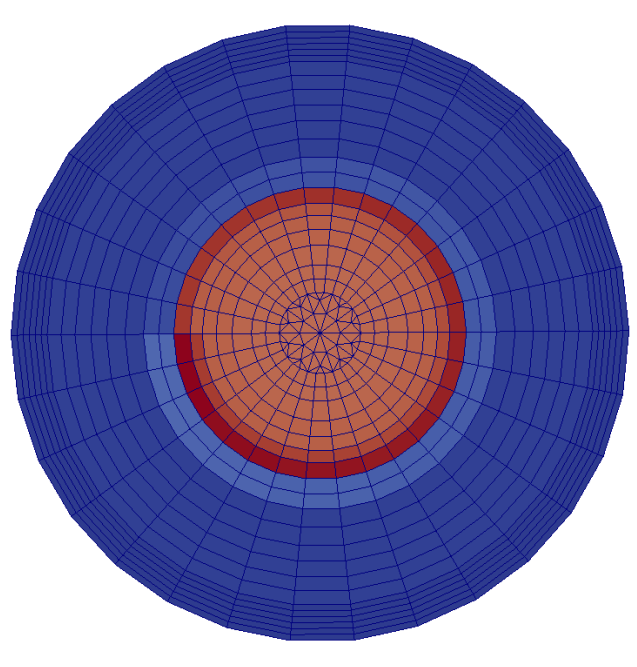

(b) Material identifier assignment within a layer at $z \approx 270 \mathrm{~cm}$ of elevation comprising the pebble bed region in the center. Note the azimuthal zoning in the outer fuel ring (darkest red) and the control rods (ring just outside the fuel region, light blue).

Figure 9: Homogenization region assignment for HTR-10. 
In addition, the meshing scripts were updated with a module to enforce volume conservation in the meshed bodies. This is a common source of error when meshing curvilinear objects with first order elements. Thus, the meshed geometry conserves the volume of each material identifier region.

\subsection{Deterministic Neutronics Solution}

The MAMMOTH reactor physics application is used as the primary tool within this benchmark exercise. MAMMOTH currently relies on external codes to provide tabulated cross sections and the FEM mesh; these are prepared using Serpent and CUBIT described in sections 3.2 and 3.3 , respectively.

The primary distinction between the Serpent and the MAMMOTH model is spatial homogenization. Spatial homogenization is the process of lumping distinct regions differing in material or flux spectrum into a single homogeneous region. Serpent provides flux-volume weighted cross sections for homogenized regions, but deploying these cross sections without corrections usually leads to unacceptably large errors caused by differences due to the presence of homogenization errors. Homogenization errors stem from the fact that homogenized regions lead to different flux solutions than the ones obtained from the originating, heterogeneous geometry: as a simple example consider a control rod smeared over the surrounding graphite; the heterogeneous configuration features a flux depression in the highly absorbing control rod region reducing the overall absorption while the homogenized region does not exhibit this characteristic. MAMMOTH is equipped with the PJFNK-SPH equivalence correction method that uses the Serpent computed reference fluxes over equivalence regions. The PJFNK-SPH method adjusts cross sections within each equivalence region via an energy group dependent SPH factor so that the MAMMOTH computed flux in this equivalence region matches the Serpent computed reference flux values. The computation of the SPH factors requires the solution of a nonlinear problem that is completed prior to the flux solution.

Within the HTR-10 model, the peripheral boronated bricks and the bottom carbon bricks pose great challenges for the Serpent code to obtaining cross section and flux tallies with reasonable statistical fidelity because few neutrons stream far enough to make collisions throughout the boronated bricks. The poor statistics lead to problems with the SPH procedure that relies on physically reasonable flux and cross section distributions. In order to obtain reasonable statistics for these zones a very large number of particle history would be needed, thus rendering the runtimes for the Monte Carlo simulation too costly. Within this 
work, we remove the boronated brick and bottom carbon brick regions from the MAMMOTH model and apply vacuum boundary conditions on all resulting external surfaces. Physically, this translates to replacing the boronated bricks with a perfect absorber, i.e. any neutron entering it is absorbed immediately regardless of its energy. Then we apply the SPH algorithm to the remaining reactor geometry using the fluxes and cross sections computed with a Serpent model that does contain the boronated and carbon brick regions. The vacuum boundary conditions reasonably approximate the neutronics behavior of the boronated bricks so that convergence of the nonlinear problem for computing the SPH factors is achieved. The result from the computed cross section sets and SPH factors is expected to be good for the following reason. Via the SPH method, we ensure that the MAMMOTH computed fluxes in each equivalence region match the Serpent computed reference fluxes. As the Serpent reference fluxes are computed using a full geometry model, the SPH factors correct for the omission of the peripheral boronated and carbon bricks. The key is that the vacuum boundary conditions that are applied in the MAMMOTH model are a reasonable approximation of the effect of the boronated bricks and hence the SPH correction applied to the regions right at the boundary are sufficiently small.

MAMMOTH relies on the Rattlesnake radiation transport application that features a variety of different angular and spatial discretization methods. Within this work only the diffusion approximation discretized with continuous FEM and the $P_{1}$ and $P_{3}$ spherical harmonics approximations of the second order transport equation discretized with continuous FEM are used for the flux solution. All three methods are suitable for moderately anisotropic angular flux distributions as encountered in PBRs. Throughout the benchmark exercise, linear Lagrange basis functions on mixed FEM meshes (triangular prisms - "wedges" in the center of the core and hexahedrons everywhere else) are used. We ensured that mesh refinement is sufficient to not affect the solution significantly by solving the problem on an additional uniformly refined mesh in the initial testing phase.

The HTR-10 benchmark exercises defined in Ref.[2, 3] require the solution of various keigenvalue problems at varying core geometries, temperatures, and rod configurations. We use MOOSE's NonlinearEigen solver with a single free power iteration for solving the keigenvalue problem; free power iterations ensure that the initial guess is sufficiently close to the fundamental mode so that the NonlinearEigen solver does not converge to a higher harmonic solution. The NonlinearEigen solver uses the preconditioned Jacobian-free NewtonKrylov with the preconditioning matrix containing only block diagonal entries and being inverted by the hypre boomeramg algebraic multigrid method. Convergence is tested based on the $L_{2}$ norm of the FEM residual; for convergence we require the norm of the residual to be smaller than $10^{-8}$ or having dropped by more than seven orders of magnitude, i.e. the 
final residual norm is smaller or equal than $10^{-7}$ times the first residual norm. 


\section{Results}

In this section we present the results obtained with Serpent and MAMMOTH for various HTR-10 calculations. We compare the results to experimentally measured values and to the benchmark values in the IRPhEP [2] and the participants from Ref. [3]. Section 4.1] deals principally with the initial criticality. In Section 4.2, we present the various calculations for the computation of the isothermal reactivity coefficients (ITC). Finally, the control rod worth calculations with the full core configuration are included in Section 4.3 .

\subsection{Benchmark Problem B1: Initial Criticality}

The eigenvalues obtained with Serpent and MAMMOTH for the initial critical core are included in Table 2. First, we note that the Serpent calculation with ENDF/B-VI based data is consistent with the IRPhEP high fidelity MCNP benchmark results. The difference of $163 \mathrm{pcm}$ can be attributed to the modeling differences discussed in Sec. 3.2 .

Serpent's and MAMMOTH's prediction of the eigenvalue in the critical configuration using ENDF/B-VII.r1 data matches the experimental value keff $=1$ very well. For this calculation we have a critical core height of $144 \mathrm{~cm}$ with the upper core conus or an equivalent surface plane of $123 \mathrm{~cm}$. The Serpent model is $23 \mathrm{pcm}$ above critical and the MAMMOTH $\mathrm{SPH}$ corrected model is $89 \mathrm{pcm}$ above critical.

Table 2: Eigenvalues computed with different codes for the initial critical configuration. (Results obtained from literature are shaded in gray.)

\begin{tabular}{ccc}
\hline Code & keff & $\begin{array}{c}\text { uncertainty } \\
\text { rel. error [pcm] }\end{array}$ \\
\hline MCNP (ENDFB-VI) [2] & 1.01190 & \pm 21 \\
Serpent (ENDFB-VI) & 1.01025 & \pm 5.1 \\
Serpent (ENDFB-VII.r1) & 1.00023 & \pm 2.3 \\
MAMMOTH TDC-SPH-Diffusion & 1.00089 & 67.3 \\
\hline
\end{tabular}

An additional validation step for the Serpent model was performed with the control rod worth measurement performed by INET. The rod measurement is reported in the IRPhEP benchmark [2] and it was conducted with a core containing 17,000 pebbles. Therefore, 110 pebbles were added to the Serpent model of the initial critical core. The rod worth 
measurement and model prediction are included in Table 3. The all-rods-out (ARO) and all-rods-in (ARI) eigenvalues are provided. The calculated control rod worth is almost identical to the measured value.

Table 3: Single Control Rod Worth for the 17k initial critical core configuration. Results obtained from literature are shaded in gray.

\begin{tabular}{ccc}
\hline Code (case) & keff & Rod worth \\
\hline Experiment & - & $1.4693 \%$ \\
Serpent (ARO) & 0.99102 & - \\
Serpent (1RI) & 0.97693 & - \\
Serpent & - & $1.4558 \%$ \\
\hline
\end{tabular}

In order to confirm the accuracy of the MAMMOTH results, the neutron production and absorption rates are compared to the Serpent computed values in the 1,443 distinct equivalence regions of the active core and reflectors. It is worth noting that these Serpent reaction rates are computed from the homogenized cross sections for each region and the flux tallies used for the SPH data. However, we expect these derived reaction rates to match the direct tallies of the reaction rates from Serpent quite well. To facilitate a comparison between MAMMOTH and Serpent reaction rates, we define the RMS and max errors as:

$$
\begin{aligned}
\text { RMS: } \varepsilon_{\mathrm{RMS}} & =\sqrt{\frac{1}{N} \sum_{j=1}^{N}\left(1-\frac{R_{j}}{L_{j}}\right)^{2}} \\
\max : \varepsilon_{\max } & =\max _{j=1, . ., N}\left|1-\frac{R_{j}}{L_{j}}\right|
\end{aligned}
$$

where $N=1,443$ and $j$ indexes the equivalence regions, $R_{j}$ and $L_{j}$ are integral reaction rates (either absorption or fission neutron production) over equivalence region $j$ computed with MAMMOTH and Serpent, respectively.

Table 4 shows that the results obtained with diffusion employing the transport cross section calculated in Serpent for the void region above the core, but without applying the SPH equivalence correction are $\sim 2,500 \mathrm{pcm}$ high due to errors emerging form discretization, homogenization, and the multi-group approximation. Similarly to the computed eigenvalue, the errors in the reaction rates, $\varepsilon_{\mathrm{RMS}}$ and $\varepsilon_{\max }$, are quite high. When Morel's tensor diffusion coefficients are employed, the solution improves and better matches the P1 and P3 solutions also provided in Table 4 
Table 4: Relative differences of eigenvalues ( $\mathrm{pcm}$ ) and reaction rates $(\%)$ between MAMMOTH and the Serpent model for the initial critical core. MAMMOTH is executed using different options including SPH corrected diffusion with and without tensor diffusion coefficients (TDC) as well as $P_{1}$ and $P_{3}$ options. We observe that with SPH correction MAMMOTH computes extremely accurate eigenvalues, as well as absorption and generation rate distributions. This implies that the homogenization error dominates other sources of uncertainty.

\begin{tabular}{ccccccccc}
\hline \multirow{2}{*}{ Solution } & \multicolumn{2}{c}{ Eigenvalue } & & \multicolumn{2}{c}{ Absorption } & & \multicolumn{2}{c}{ Generation } \\
\cline { 2 - 3 } & keff & $\Delta(\mathrm{pcm})$ & & RMS & $\max$ & & RMS & $\max$ \\
\hline Diffusion & 1.02544 & 2520.8 & & 21.5 & 153 & & 36 & 41.1 \\
TDC-Diffusion & 1.03475 & 3451.3 & & 17.4 & 117.9 & & 34.7 & 41.7 \\
P1 & 1.03706 & 3682.3 & & 20.7 & 144.6 & & 2.849 & 6.53 \\
P3 & 1.04053 & 4029.0 & & 19.6 & 134.7 & & 2.976 & 7.12 \\
SPH-Diffusion & 1.00090 & 67.3 & & 0.16 & 0.4 & & 0.062 & 0.11 \\
TDC-SPH-Diffusion & 1.00089 & 66.1 & & 0.16 & 0.4 & & 0.059 & 0.11 \\
\hline
\end{tabular}

This leads us to conclude that the cross section homogenization carries a 4,000 pcm bias; the magnitude of the bias is consistent with the expected error originating from the control rod homogenization in the top reflector region. When the SPH equivalence is applied to the cross sections, we closely match the reference solution from Serpent. The error in the eigenvalue is less than $100 \mathrm{pcm}$ and the reaction rates are within $0.5 \%$ of the Monte Carlo result. The TDC-SPH work-flow originates from the Transient Test Reactor modeling and simulation tasks performed at INL [14]. The use of TDC-SPH for this PBR only marginally improves the solution. Furthermore, the use of TDCs would also have a smaller impact in the full core configuration, where the streaming zone above the active core is smaller. Therefore, a direct SPH correction will be used in the rest of the calculations, thus bypassing the generation of TDCs. A flux solution through the critical core's center line is shown in Figure 10. The effect of the upper cavity is clearly visible as the flux is almost flat within this region. The thermal flux peaks in the bottom conus are also visible.

\subsection{Benchmark Problem B2: Temperature Coefficient of Reactivity}

The purpose of the $\mathrm{B} 2$ problem is to compute the eigenvalues for the full core configuration at three uniform core temperatures: 20,120 , and $250^{\circ} \mathrm{C}$. The Serpent, MAMMOTH SPH- 


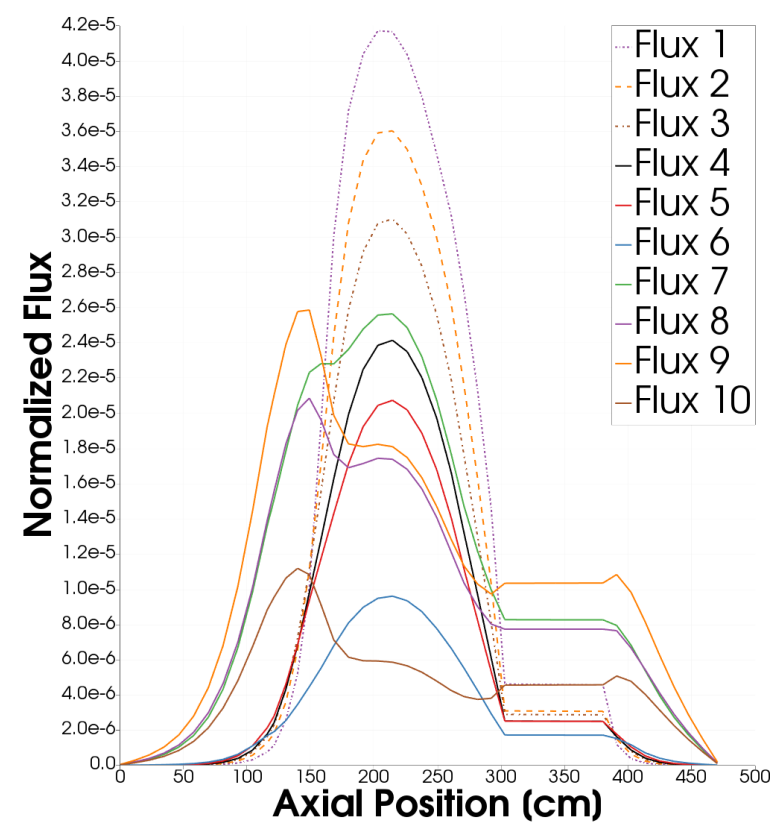

Figure 10: Flux distribution in the critical core (axial centerline).

Diffusion and the results from Ref. [3] are shown in Table 5. The isothermal temperature coefficient was computed and is shown in Table 6 since all participants used cross section data based on JEF-1 and ENDF/B-V. We observe that the Serpent and MAMMOTH results agree well with the German VSOP results. The German VSOP [26] package, which is also used by South Africa (SA) and China, relies on a variety of deterministic radiation transport codes to compute homogenized few group cross sections. While the difference in results obtained with the same code is surprising, it emphasizes the effect of modeling approximations adopted by different analysts. This discrepancy was already observed and noted during the preparation of the benchmark document, Ref. [3], but no explanation was provided. The temperature feedback in this fresh core is from both Doppler broadening of ${ }^{238} \mathrm{U}$ and the spectral shifting from up-scattering. The latter shifts the neutron energy spectrum away from the $1 / \mathrm{v}$ region, thus effectively reducing the fission rate in ${ }^{235} \mathrm{U}$. We ran the Serpent models with ENDF/B-VI and ENDF/B-VII.r0 and obtained similar results.

The neutron production and absorption rates obtained with MAMMOTH are compared to the Serpent values in the RMS and max relative errors defined in Eq. 4 for the full core configuration in Table 7. We observe a dramatic improvement if the SPH equivalence correction is applied that is consistent with the critical core results. The difference in the eigenvalue is reduced from $\sim 2,500 \mathrm{pcm}$ to less than $100 \mathrm{pcm}$. In addition, the neutron 
Table 5: Eigenvalues at three temperatures computed with various codes for the full core. Results obtained from literature are shaded in gray. The MAMMOTH computed eigenvalues are obtained with SPH corrected diffusion.

\begin{tabular}{cccc}
\hline Code & $\begin{array}{c}\mathrm{B} 21 \\
\left(20 \text { or } 27^{\circ} \mathrm{C}\right)\end{array}$ & $\begin{array}{c}\mathrm{B} 22 \\
\left(120^{\circ} \mathrm{C}\right)\end{array}$ & $\begin{array}{c}\mathrm{B} 23 \\
\left(250{ }^{\circ} \mathrm{C}\right)\end{array}$ \\
\hline Serpent (INL) & $1.12242 \pm 13$ & $1.11068 \pm 20$ & $1.09298 \pm 14$ \\
MAMMOTH (INL) & 1.12242 & 1.11061 & 1.09249 \\
VSOP (China) & 1.1358 & 1.1262 & 1.1111 \\
MCNP4 (China) & 1.1381 & - & - \\
VSOP 2-D (Germany) & 1.1468 & 1.1334 & 1.1160 \\
VSOP 3-D (Germany) & 1.1368 & 1.1232 & 1.1054 \\
TRIPOLI4 (France) & 1.1474 & - & \\
VSOP_PBMR (SA) & 1.1286 & 1.1196 & 1.1047 \\
\hline
\end{tabular}

absorption and generation rates are reduced to less than $0.5 \%$ compared to the Monte Carlo reference. 
Table 6: Isothermal Temperature Coefficient of Reactivity computed as slope of the secant over the stated temperature range for the full core. Results obtained from literature are shaded in gray. The MAMMOTH computed eigenvalues are obtained with SPH corrected diffusion.

\begin{tabular}{ccc}
\hline Code & $\begin{array}{c}20-120{ }^{\circ} \mathrm{C} \\
{\left[\mathrm{pcm} /{ }^{\circ} \mathrm{C}\right]}\end{array}$ & $\begin{array}{c}120-250{ }^{\circ} \mathrm{C} \\
{\left[\mathrm{pcm} /{ }^{\circ} \mathrm{C}\right]}\end{array}$ \\
\hline Serpent (INL) & -9.33 & -11.28 \\
MAMMOTH (INL) & -10.31 & -11.49 \\
VSOP (China) & -7.51 & -9.28 \\
VSOP 2-D (Germany) & -10.31 & -10.58 \\
VSOP 3-D (Germany) & -10.65 & -11.03 \\
VSOP PBMR (SA) & -7.16 & -9.25 \\
\hline
\end{tabular}

Table 7: Relative differences of eigenvalues ( $\mathrm{pcm}$ ) and reaction rates $(\%)$ between MAMMOTH and the Serpent model for the full core under isothermal conditions. MAMMOTH's diffusion options with and without SPH corrections are used. It is apparent that with SPH correction highly accurate results are obtained underpinning the importance of SPH for removing the homogenization error.

\begin{tabular}{|c|c|c|c|c|c|c|}
\hline \multirow{2}{*}{ Temperature (K) } & \multicolumn{2}{|c|}{ Eigenvalue } & \multicolumn{2}{|c|}{ Absorption } & \multicolumn{2}{|c|}{ Generation } \\
\hline & keff & $\Delta(\mathrm{pcm})$ & RMS & $\max$ & RMS & $\max$ \\
\hline \multicolumn{7}{|c|}{ Diffusion } \\
\hline 300 & 1.15096 & 2542.7 & 20.6 & 136.19 & 6.61 & 13.3 \\
\hline 393 & 1.13793 & 2453.5 & 20.5 & 133.59 & 6.68 & 13.2 \\
\hline 523 & 1.12009 & 2480.6 & 20.2 & 129.51 & 6.79 & 13.8 \\
\hline \multicolumn{7}{|c|}{ SPH corrected Diffusion } \\
\hline 300 & 1.12347 & 93.9 & 0.2 & 0.5 & 0.1 & 0.19 \\
\hline 393 & 1.11061 & -5.9 & 0.14 & 0.33 & 0.06 & 0.13 \\
\hline 523 & 1.09249 & -44.5 & 0.05 & 0.12 & 0.02 & 0.05 \\
\hline
\end{tabular}




\subsection{Benchmark Problem B3: Control Rod Worth}

The B3 problem in the benchmark is similar to the control rod worth calculation performed in Section 4.1 with the exception that it requires the computation of all rods out and one rod in for the full core configuration. The Serpent, MAMMOTH SPH-Diffusion and the results from Ref. [3] are shown in Table 8, In the all rods in case our results are within the statistical spread from the various participants, but for the one rod case we appear to be in the upper end of the distribution, but, again, consistent with the German VSOP results. Some of the uncertainty arises from the absence of a strict definition of rod worth from the source of the reported values, $\rho$ versus $\Delta \rho$.

Table 8: Control Rod Worth [\%] for the full core configuration; in case B31 the difference in reactivity between all rods out and all rods in is reported while for the B32 case the difference between all rods out and one rod in is reported. Results obtained from literature are shaded in gray.

\begin{tabular}{ccc}
\hline Code & B31 (all rods) & B32 (one rod) \\
\hline Experiment $^{1}$ & - & 1.4693 \\
Serpent (INL) & $14.40 \mid 14.99$ & $1.56 \mid 1.41$ \\
MAMMOTH (INL) & $14.42 \mid 15.00$ & $1.54 \mid 1.39$ \\
VSOP (China) $^{2}$ & 14.46 & 1.28 \\
MCNP4 (China) & 15.31 & 1.34 \\
VSOP (Germany) & 15.73 & 1.48 \\
TRIPOLI4 (France) & 13.44 & 1.31 \\
\hline
\end{tabular}

${ }^{1}$ Measured with the smaller 17,000 pebble core

${ }^{2}$ Reported values are $\rho \mid \Delta \rho$

The neutron production and absorption rates obtained with MAMMOTH are compared with Serpent results full core configuration depicted in Fig. 9 and RMS and maximum relative errors are computed. The sensitivity to the control rod position is quite pronounced without SPH equivalence. The largest difference in the eigenvalue for the SPH-Diffusion occurs in the single rod insertion case, which corresponds to the most difficult configuration due to the asymmetry in the flux distribution. Nevertheless, the eigenvalue is within $\sim 120$ $\mathrm{pcm}$ of the reference Monte Carlo value. The maximum error in the absorption rate is also low, within $0.536 \%$ compared to the reference case.

A diametric scalar flux distribution for all energy groups for the one rod in case is shown in Figure 11. The line-out intersects the inserted rod to the left of the reactor midpoint at 200 
Table 9: Relative difference of MAMMOTH computed eigenvalues ( $\mathrm{pcm}$ ) and reaction rates $(\%)$ with the Serpent solution for the full core with different rod configurations (ARO - all rods out, ARI - all rods in, and 1RI - one rod in).

\begin{tabular}{|c|c|c|c|c|c|c|}
\hline \multirow{2}{*}{ Configuration } & \multicolumn{2}{|c|}{ Eigenvalue } & \multicolumn{2}{|c|}{ Absorption } & \multicolumn{2}{|c|}{ Generation } \\
\hline & keff & $\Delta(\mathrm{pcm})$ & RMS & $\max$ & RMS & $\max$ \\
\hline \multicolumn{7}{|c|}{ Diffusion } \\
\hline ARO & 1.15096 & 2542.7 & 20.6 & 136.19 & 6.6 & 13.3 \\
\hline ARI & 0.97094 & 1061.3 & 24.6 & 158.07 & 10.4 & 21.6 \\
\hline $1 \mathrm{RI}$ & 1.13118 & 2378.5 & 21.3 & 161.9 & 6.97 & 14.6 \\
\hline \multicolumn{7}{|c|}{ SPH corrected Diffusion } \\
\hline ARO & $1.12 \overline{347}$ & 93.9 & 0.2 & 0.5 & 0.1 & 0.19 \\
\hline ARI & 0.96145 & 74.1 & 0.2 & 0.51 & 0.09 & 0.16 \\
\hline $1 \mathrm{RI}$ & 1.10622 & 119.4 & 0.2 & 0.54 & 0.1 & 0.22 \\
\hline
\end{tabular}

$\mathrm{cm}$. We observe that the more thermal fluxes are depressed in the vicinity of the control rod at diametric distance of $\approx 100 \mathrm{~cm}$. The peak for all flux groups is shifted significantly to the right from the geometrical center at $190 \mathrm{~cm}$.

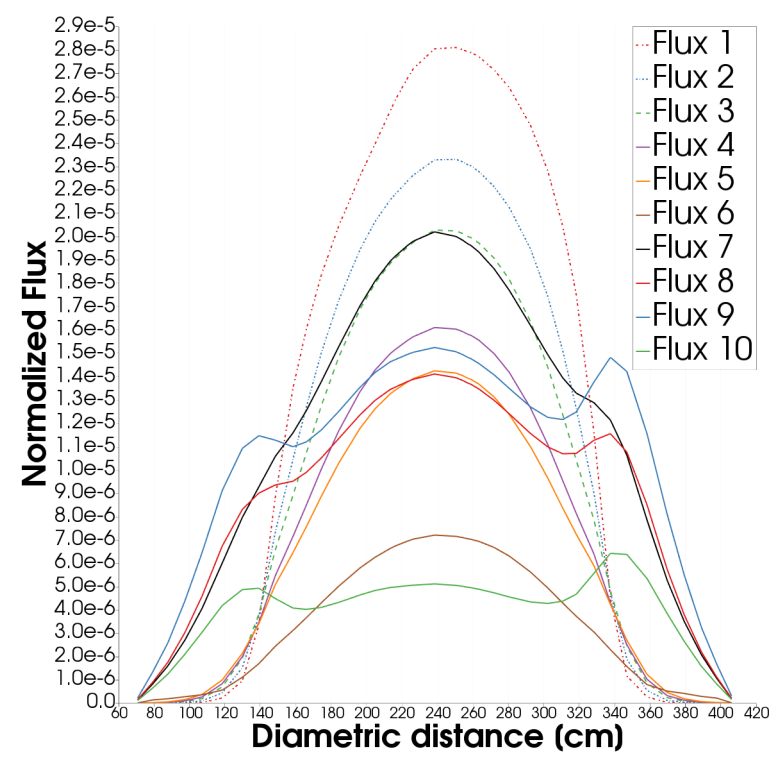

Figure 11: Diametric flux distribution for the one-rod-in configuration through the control rod region. 
A three-dimensional rendering of the scalar flux in group 10 for the one rod in case is provided in Fig. 12, the outline of the reactor core and the whole HT-10 model are included in Fig. 12. The depression of the flux around the inserted control rod positioned just outside the core region is clearly visible.

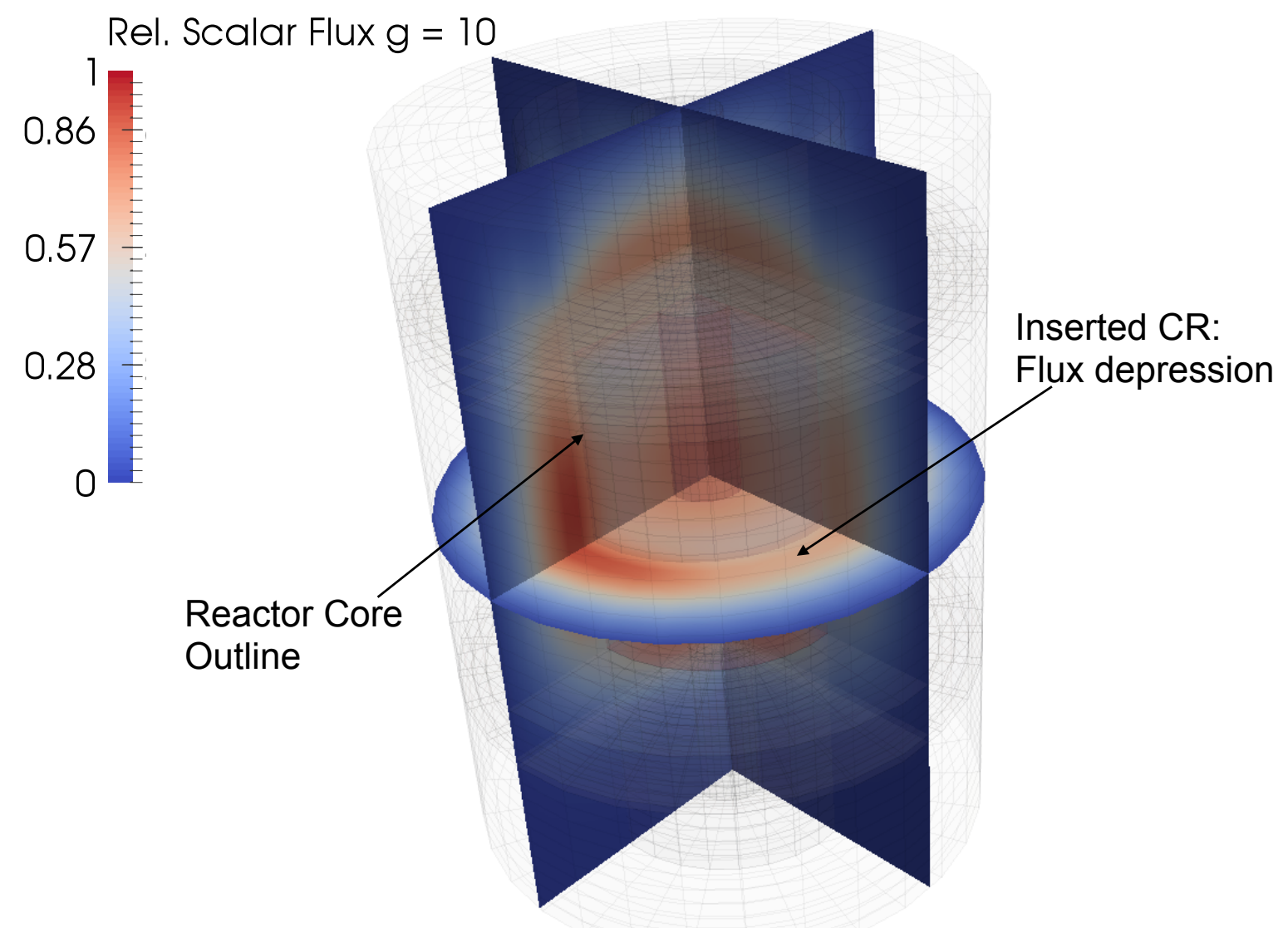

Figure 12: Rendering of the scalar flux in group 10 (most thermal flux) for the one-rod in configuration. The depression of the flux around the inserted control rod positioned just outside the core region is clearly visible.

In Fig. 13 we show the most thermal flux distribution in a slice at $z=270 \mathrm{~cm}$ (core region) for the 1RI, ARI, and ARO configurations along with line-out plots the flux along the line colored in light green that intersects the single inserted control rod in the 1RI configuration. We observe symmetric solutions for the ARI and ARO cases and an asymmetric solution 
driven by the single inserted control rod for the 1RI configuration. The thermal peak at the reflector interface is present for the $1 \mathrm{RO}$ and ARO configurations (interestingly also present along the line-out for the 1RI configuration) but absent for the ARO configuration because the inserted control rods reduce the thermal neutron population at the core-reflector interface.
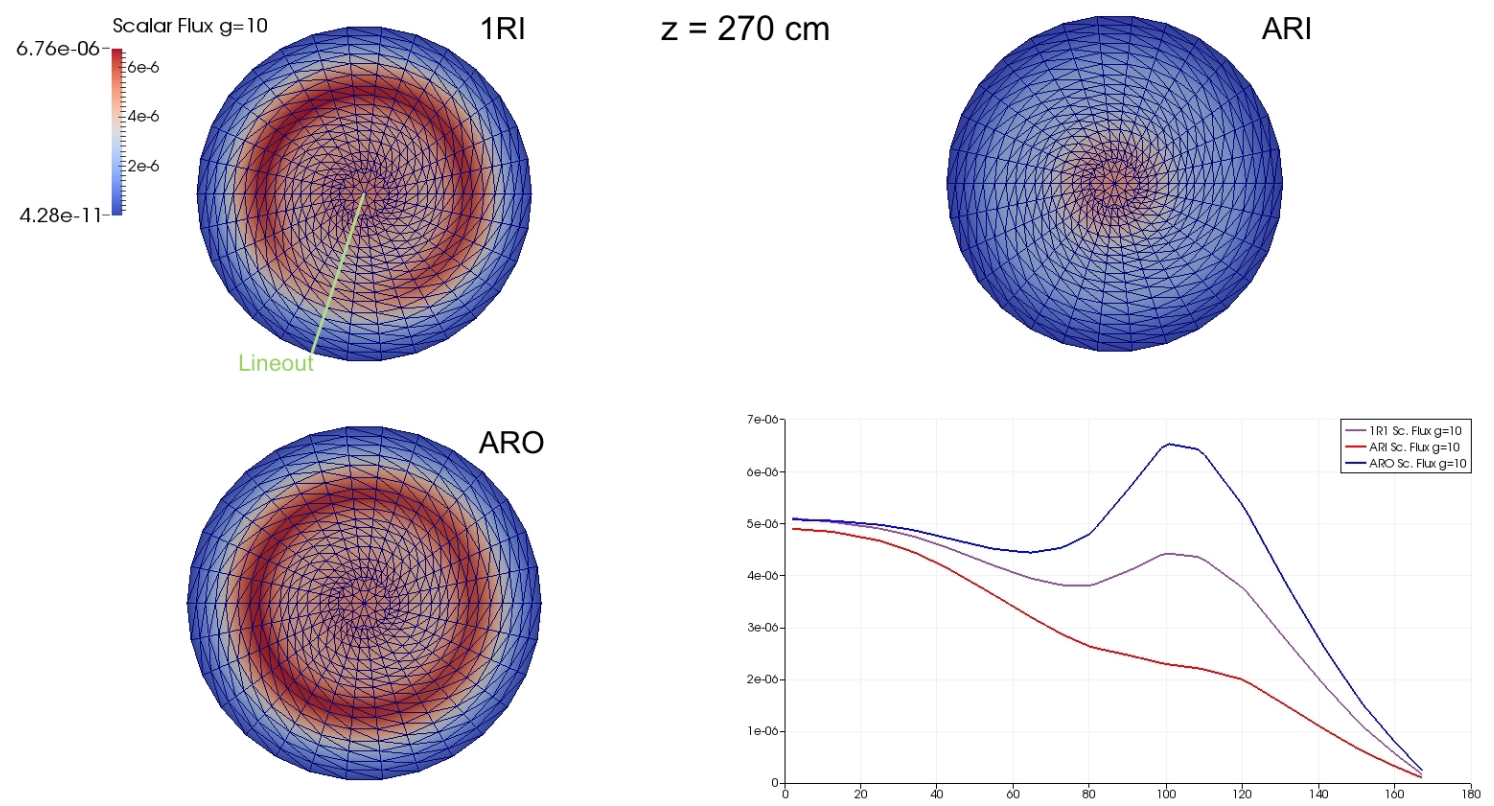

Figure 13: Rendering of the scalar fluxes in group 10 at $z=270 \mathrm{~cm}$ (lower core level) for all three rod configurations: one rod in (1RI), all rods in (ARI), and all rods out (ARO) along with traces of the scalar fluxes along a line intersecting the inserted control rod in the 1RI configuration. We observe symmetric solutions for the ARI and ARO cases and an asymmetric solution driven by the single inserted control rod for the 1RI configuration. The thermal peak at the reflector interface is present for the 1RO and ARO configurations (interestingly also present along the line-out for the 1RI configuration) but absent for the ARO configuration because the inserted control rods reduce the thermal neutron population at the core-reflector interface. 


\section{Conclusion}

We successfully developed high fidelity Monte Carlo Serpent models of the HTR-10 critical and full core configurations. The models include both a random discrete distribution of TRISO particles in the pebbles and a random pebble distribution in the pebble bed core. The Serpent results match very well the critical and control rod worth measurements provided in the International Reactor Physics Experiment Evaluation Project (IRPhEP) report with the ENDF/B-VII.r1 data-set.

We used the Serpent model to prepare 10 coarse energy group cross sections and flux tallies for the MAMMOTH Reactor Physics MOOSE application. The report shows that MAMMOTH can reproduce the Monte Carlo solution obtained with Serpent for the HTR10 reactor with the use of the PJFNK-SPH equivalence.

In addition, a set of exercises from the International Atomic Energy Agency (IAEA) HTR10 benchmark in IAEA-TECDOC-1382 were conducted. The exercises include the B1 (critical), B2 (temperature coefficient) and B3 (control rod worth). The results for the critical core are excellent. Our temperature coefficient calculation is consistent with the VSOP results generated by the German participants. The Chinese and South African VSOP models produce lower temperature coefficients. This was noted in the original IAEA benchmark, but no explanation was provided. The control rod worth calculation for all rods is consistent with the results from benchmark participants. The single rod worth calculation for the full core configuration shows that our results are close to the upper end of the reported values, but again consistent with the German VSOP results. The fact that we match well the actual experimental value for the single control rod worth with the 17,000 pebble core reinforces the accuracy of our predictions.

In all cases the MAMMOTH results are within $120 \mathrm{pcm}$ of the Serpent Monte Carlo reference calculation. The maximum errors in neutron absorption and generation rates are within $0.536 \%$ and $0.215 \%$ from the Serpent Monte Carlo reference calculation, respectively. 


\section{Future Work}

Idaho National Laboratory has developed a number of MOOSE-based applications to model and simulate a variety of interconnected regimes of physics including fuel performance in BISON [17], micro-scale phenomena in MARMOT [27], radiation damage in MAGPIE [28], porous flow and heat transfer in Pronghorn [5, 6], primary and secondary reactor system behavior in Relap-7 [29], radiation transport in Rattlesnake [16], and reactor physics in MAMMOTH [1] among others.

The unique feature of these MOOSE-based applications is that they are all built on the same FEM framework which allows seamless multiphysics coupling. Furthermore, MOOSE supports conservative solution projections between all MOOSE-based applications. This feature guarantees the strict conservation of important physical quantities, like energy, between applications. MOOSE also supports solutions to different sets of equations on different subdomains that may vary in dimensionality, and bridging of different length and time scales via the multi-app system. The MOOSE herd provides a significant leap of the multiphysics capabilities available for engineering analysis.

After the successful completion of the stand-alone neutronics analysis of a pebble bed reactor documented in this report, our next step will be focused on the addition of other important phenomena fundamental to PBR analysis. Coupling to the Pronghorn thermalfluids application will enable multiphysics studies of the steady state coupled core behavior.

The following is a list of potential engineering applications for MAMMOTH coupled to other MOOSE based tools:

- Plant safety analysis including thermal-fluids and accident analysis (reactivity and thermal transients) [MAMMOTH, Pronghorn]

- fuel performance and fission product behavior during normal and accident conditions [MAMMOTH, BISON, MAGPIE]

- graphite performance and graphite damage studies [MAMMOTH, MARMOT, MAGPIE] 


\section{References}

[1] F. N. Gleicher, J. Ortensi, et. al. The coupling of the neutron transport application RATTLESNAKE to the fuels performance application bison. In International Conference on Reactor Physics (PHYSOR 2014), Kyoto, Japan, May 2014.

[2] Evaluation of the initial critical configuration of the HTR-10 pebble-bed reactor. Technical Report HTR10-GCR-RESR-001, International Reactor Physics Experiment Evaluation Project, 2006.

[3] Evaluation of high temperature gas cooled reactor performance:benchmark analysis related to initial testing of the httr and htr-10. Technical Report IAEA-TECDOC1382, International Reactor Physics Experiment Evaluation Project, 2003.

[4] Yaqi Wang, Sebastian Schunert, and Vincent Laboure. Rattlesnake Theory Manual. INL, 2017.

[5] L. Zou, J.W. Peterson, A.J. Novak, R. Martineau, and H.D. Gougar. Stabilized finite element formulation for thermally-driven porous media flows in pronghorn. In ANS Annual Summer Meeting 2018, Driving the Future of Nuclear Technology. ANS.

[6] H. Park, D. A. Knoll, D. R. Gaston, and R. C. Martineau. Tightly coupled multiphysics algorithms for pebble bed reactors. Nuclear Science and Engineering, 166(2):118-133, 2010.

[7] J.Cogliati and A. Ougouag. Pebbles operation and theory manual. Technical Report INL/EXT-10-19305, r1, Idaho National Laboratory, 2010.

[8] J. Leppänen,. Development of a New Monte Carlo Reactor Physics Code. PhD thesis, Helsinki University of Technology, 2007.

[9] Randy Morris. Cubit 15.0 user documentation. Technical report, ETI, UT, 2014.

[10] J. Ortensi. An Earthquake Transient Method for Pebble-Bed Reactors and a Fuel Temperature Model for TRISO Fueled Reactors. PhD thesis, Idaho State University, 2009.

[11] J.J. Cogliati, A.M. Ougouag, and J. Ortensi. Survey of dust production in pebble bed reactor cores. Nuc. Eng. Deign., 241:2364, 2011. 
[12] J. Ortensi, J.J. Cogliati, M.A. Pope, J.D. Bess, R. M. Ferrer, A.A. Bingham, and A.M. Ougouag. Deterministic modeling of the high temperature test reactor. Research Report INL/EXT-10-18969, Idaho National Laboratory.

[13] J. Ortensi, A. Alberti, Y. Wang, M.D. DeHart, F.N. Gleicher, S. Schunert, and T.S. Palrmer. Methodologies and requirements for the generation of physics data inputs to mammoth transient simulations in support of the transient reactor test facility. Technical Report NL/LTD-15-36265, Idaho National Laboratory, September 2015.

[14] J. Ortensi, B. Baker, S. Schunert, Y. Wang, F.N. Gleicher, and M.D. DeHart. Updates to the Generation of Physics Data Inputs for MAMMOTH Simulations of the Transient Reactor Test Facility - FY2016. Technical Report INL/EXT-16-39120, Idaho National Laboratory, June 2016.

[15] Derek R. Gaston, Cody J. Permann, John W. Peterson, Andrew E. Slaughter, David Andrs, Yaqi Wang, Michael P. Short, Danielle M. Perez, Michael R. Tonks, Javier Ortensi, Ling Zou, and Richard C. Martineau. Physics-based multiscale coupling for full core nuclear reactor simulation. Annals of Nuclear Energy, 84:45-54, 2015.

[16] Y. Wang. Nonlinear diffusion acceleration for the multigroup transport equation discretized with sn and continuous fem with rattlesnake. In Proceedings to the International Conference on Mathematics, Computational Methods \& Reactor Physics (M\&C 2013), Sun Valley, Idaho, USA, May 2013.

[17] R.L. Williamson et al. Multidimensional Multi-physics Simulation of Nuclear Fuel Behavior. Jou. Nucl. Mat., 423(149-163), 2012.

[18] James J. Duderstadt and Louis J. Hamilton. Nuclear Reactor Analysis. John Wiley \& Sons, Inc.

[19] J. Morel. A non-local tensor diffusion theory. Research Report LA-UR-07-5257, Los Alamos National Laboratory.

[20] T.J. Trahan and E.W. Larsen. 3-d anisotropic neutron diffusion in optically thick media with optically thin channels. In Proc. Int. Conf. on Mathematics and Computational Methods Applied in Nuclear Science and Engineering (MC 2011). ANS.

[21] Z. Liu, K. Smith, and B. Forget. A cumulative migration method for computing rigorous transport cross sections and diffusion coefficients for lwr lattices with monte carlo. In Physics of Reactors 2016 (PHYSOR 2016): Unifying Theory and Experiments in the 21 st Century. ANS. 
[22] A. Hébert, G. Mathonniere. Development of a third generation superhomogenization method for the homogenization of pressurized water reactor assembly. Nuc. Sci. Eng., 2:115, 1993.

[23] J. Ortensi, Y. Wang, A. Laurier, S. Schunert, A. Hébert, and M.D. DeHart. A newton solution for the superhomogenization method: The pjfnk-sph. Annals of Nuclear Energy, 111:579-594, January 2018.

[24] Y. Wang, J. Ortensi, S. Schunert, and V. Laboure. A pebble tracking transport algorithm for pebble bed reactor analysis. In PHYSOR 2018: Reactor Physics paving the way towards more efficient systems, Cancun, Mexico, April 2018.

[25] Victor R. Yarberry Larry A. Schoof. EXODUS II: A Finite Element Data Model, SAND92-2137. Technical report, Sandia National Laboratory, December 1995.

[26] E. Teuchert et al. V.S.O.P (94) Computer Code System for Reactor Physics and Fuel Cycle Simulation. Research Report Jülich-2897, Forschungszentrum Jülich, 1994.

[27] D. Schwen, L. K. Aagesen, Y. Zhang, K. Ahmed, B. Beeler, M. R. Tonks, D. A. Andersson, and C. Matthews. Assessment of marmot release 1.2: A mesoscale fuel performance code. Research Report INL/EXT-16-39975, Idaho National Laboratory.

[28] S. Schunert, D. Schwen, P. Ghassemi, B. Baker, A. Zabriskie, J. Ortensi, Y. Wang, F. Gleicher, M. DeHart, and R. Martineau. Heat source characterization in a treat fuel particle using coupled neutronics binary collision monte-carlo calculations. In $M \& C$ 2017 International Conference on Mathematics and Computational Methods Applied to Nuclear Science \& Technology. American Nuclear Society.

[29] D. Andrs et al. RELAP-7 Level 2 Milestone Report: Demonstration of a Steady State Single Phase PWR Simulation with RELAP-7. Technical Report INL/EXT-12-25924, Idaho National Laboratory, 2012. 